\title{
Development of friction free controller for electro-hydrostatic actuator using feedback modulator and disturbance observer
}

\author{
Sho Sakaino ${ }^{*+}{ }^{-10}$ and Toshiaki Tsuji ${ }^{\dagger}$
}

\begin{abstract}
Hydraulic actuators have high power-to-weight ratios, making them suitable for high-power robotic applications such as in walking robots and construction machines. However, large frictional forces in hydraulic actuators, rotary hydraulic actuators in particular, degrade the control performance. To suppress frictional forces and increase robustness against modeling errors, this study considered the integration of feedback modulators (with minimum control inputs exceeding static frictional forces) with disturbance observers. In the proposed controller, nonlinear static frictional forces are suppressed by the feedback modulators and linear disturbances are suppressed by the disturbance observers. The validity was experimentally verified in this study.
\end{abstract}

Keywords: Hydraulic system, Friction compensation, Electro-hydrostatic actuator, Disturbance observer, Feedback modulator

\section{Background}

Recent trends in robotics require actuators with high power-to-weight ratios. For example, robots designed to support human movement must be light for safety and to enable standing and walking, and machines used for construction must have sufficient force to manipulate large objects. Hydraulic actuators are commonly used in these applications because of their power-to-weight ratios and their remote driving units (i.e., power sources, electric motors, servo units, and oil tanks).

Two types of circuits are commonly used to control hydraulic actuators: open and closed. In open circuits, power generated by a motor is reduced and regulated by a valve and is transmitted to a hydraulic actuator. Much of the power is dissipated in the valve, resulting in lower efficiency and larger system designs. Figure 1 shows a schematic of an open hydraulic circuit. In closed circuits (known as electro-hydrostatic actuators or EHAs) a servomotor generates and directly controls the power.

\footnotetext{
*Correspondence: sakaino@mail.saitama-u.ac.jp

'Sho Sakaino and Toshiaki Tsuji contributed equally to this work Graduate School of Science and Engineering, Saitama University, 255, Shimo-okubo, Sakura-ku, Saitama, Japan
}

Figure 2 shows a schematic of a closed hydraulic circuit (EHA). Closed hydraulic circuits offer three advantages: (1) the systems are simple and small; (2) the efficiency is high; (3) valve-related nonlinearities are omitted. However, there is a drawback in EHAs; the control performance of EHAs is worse than that of open hydraulic circuits. Therefore, control performance of EHAs is improved in this study.

Given that many of the inherent nonlinearities are omitted in EHAs, the system dynamics can be well characterized by a linear model [1]. Hence, complicated controllers such as adaptive controllers [2], sliding mode controllers [3], and fuzzy controllers [4] are not necessary, while those are often implemented in hydraulic systems. However, actuator efficiencies still introduce nonlinear effects. Figure 3 shows the volume and torque efficiencies for the S-380 actuator made by Eaton Industries Ltd., and used in this study [5]. Note that actuator efficiencies are dependent on the angular velocity. Furthermore, efficiencies in the low speed domain are not given; high oil leakage in the low-speed domain poses a challenge in the accurate estimation of efficiencies. The effects of oil leakage are characterized as static friction because the applied force decreases in the low speed 


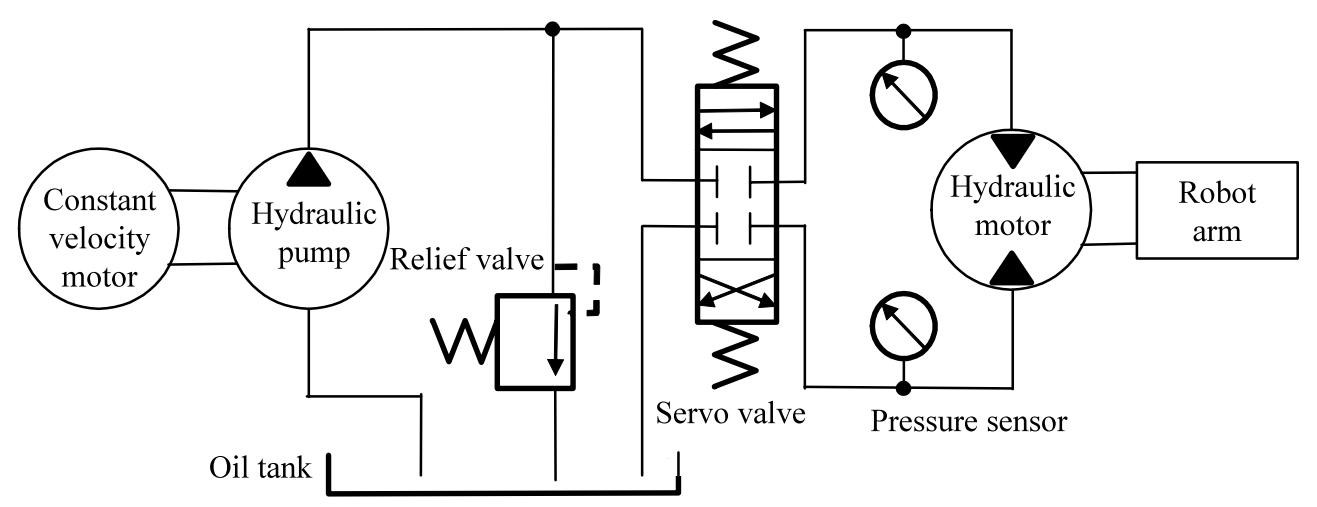

Fig. 1 Open hydraulic circuit

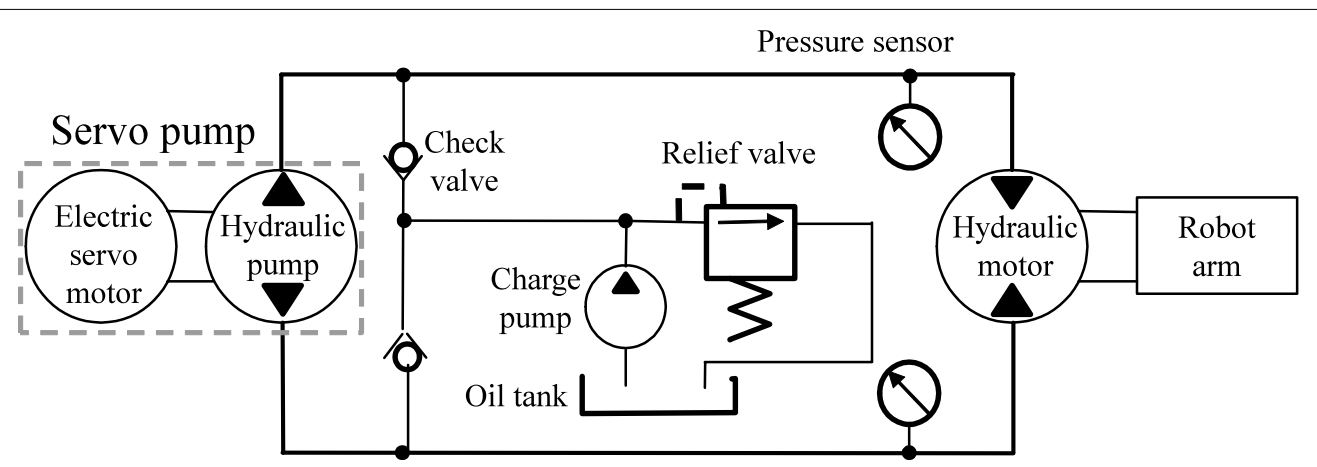

Fig. 2 Closed hydraulic circuit (electro-hydrostatic actuator)

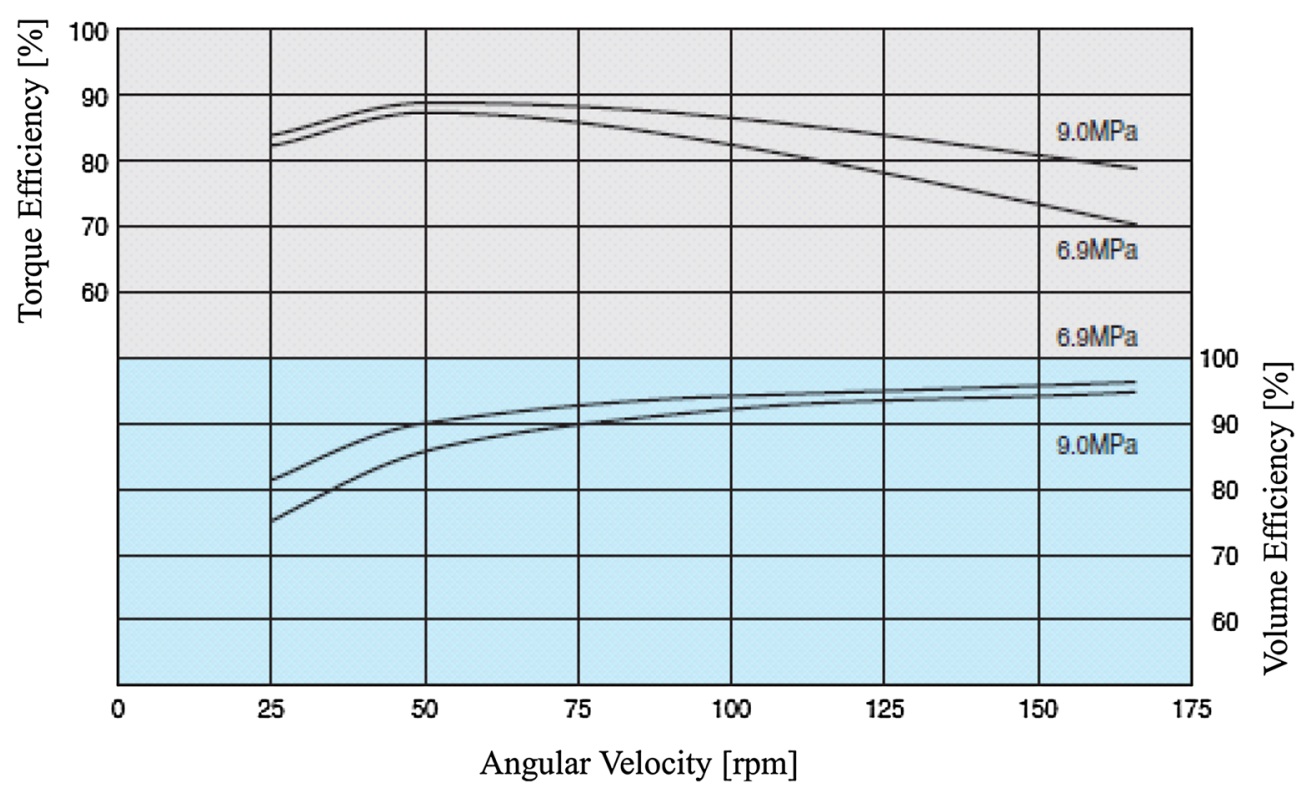

Fig. 3 Actuator efficiency 
domain. Therefore, the suppression of the frictional effect is essential for fine control.

Prior research purports that the frictional effect can be suppressed using adaptive control $[6,7]$; however, adaptive control requires significant tuning parameters and is quite difficult to implement. For practical implementation, hydraulic controllers should be easy to design. Feedback modulators (FMs) have been successfully implemented in a hydraulic system to suppress static friction [8]. When an FM's minimum force is set to exceed the maximum static frictional force, systems are then free from static friction. However, FMs cannot suppress disturbances caused by gravity, viscosity, modeling errors, etc. Disturbance observers (DOBs) can instead be used to suppress these types of disturbances [9-11]. However, DOBs generate residual oscillation if the static friction is large. Yao et al. proposed an extended state observer to compensate for disturbances in hydraulic systems [12]. However, the controller was designed for open hydraulic circuits. To address these issues, we proposed a new controller that integrates FMs and DOBs [13]. In the previously proposed controller, FMs are only activated in the low-speed domain and DOB compensation value updates are concurrently suspended. The previously proposed controller offers three advantages: (1) in the low-speed domain, the FMs eliminate frictional effects; (2) in other speed domains, DOBs operate as normal; (3) the design is simple and does not require strict parameter tuning.

In the previous study [13], the proposed controller was implemented in an open hydraulic circuit. The proposed controller's effectiveness for EHAs was unconfirmed because of the different dynamic characteristics of open and closed circuits. In EHAs, the

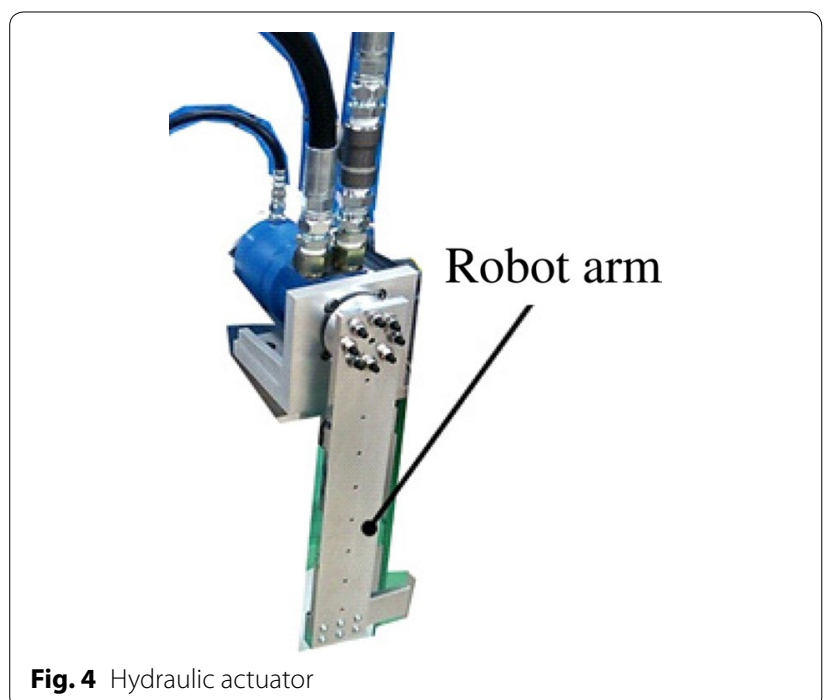

hydraulic pumps introduce additional static friction, resulting in greater frictional force. We implemented the proposed controller in an EHA and verified its effectiveness. To overcome the shortcomings in prior studies, we compared the proposed controller with other friction compensators, including dither signals. Dither signals have previously been shown to eliminate frictional effects [14].

\section{Modeling}

\section{Hydraulic actuator and robot arm}

Figures 4 and 5 show the actual hydraulic actuator/ robot arm (Eaton Industries, Ltd., S-380) and a force model of the hydraulic actuator/robot arm used in this study, respectively. In this paper, the hydraulic actuator and robot arm are referred to as load-side. The angular response of the hydraulic actuator was obtained using an optical encoder with 17 bit resolution in every $1 \mathrm{~ms}$. The dynamics of the load-side is given as follows:

$$
m_{L} \ddot{\theta}_{L}^{r e s}=\eta_{t L} \frac{D_{L}}{2 \pi} p^{r e s}
$$

where $m, \theta, D, p$, are inertia, angle, displacement, and pressure difference between the input and output ports, respectively. $\eta_{t L}$ is torque efficiency of the hydraulic actuator. Response variables are denoted by the superscript res. Variables with a subscript $L$ represent the load-side.

\section{Hydraulic system}

Figure 6 depicts the complete hydraulic system used in this study. An electric servomotor drives a hydraulic pump (Eaton Industries, Ltd., MA-03) that then supplies oil to the hydraulic actuator. In this paper, the hydraulic pump and the servomotor are referred to as motor-side. The equation of motion of the motor-side is given as follows:

$$
m_{M} \ddot{\theta}_{M}^{r e s}=\eta_{t M} K_{t} I_{M}^{r e f}-\frac{D_{M}}{2 \pi} p^{r e s}
$$

where $K_{t}$ and $I_{M}^{r e f}$ are a servomotor's torque constant and current reference, respectively. Variables with a subscript $M$ represent the motor-side. $\eta_{t M}$ is the torque efficiency of the hydraulic pump.

By substituting (1) into (2), the following dynamic relationship is obtained.

$$
m_{M} \ddot{\theta}_{M}^{r e s}=\eta_{t M} K_{t} I_{M}^{r e f}-\frac{1}{\eta_{t L}} \frac{D_{M}}{D_{L}} m_{L} \ddot{\theta}_{L}^{r e s}
$$

Since the oil flow is continuous, the following equation is satisfied.

$$
\frac{D_{M}}{2 \pi} \dot{\theta}_{M}^{r e s}=\frac{D_{L}}{2 \pi} \dot{\theta}_{L}^{r e s}
$$




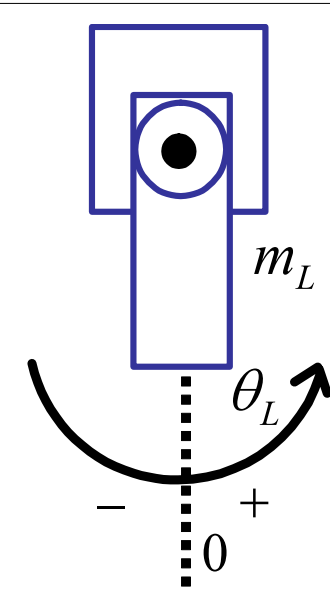

Fig. 5 Robot model

Finally, the dynamics can be calculated from (3) and (4):

$$
\begin{aligned}
\left(n^{2} \eta_{t L} m_{M}+m_{L}\right) \ddot{\theta}_{L} & =n \eta_{t M} \eta_{t L} K_{t} I_{M}^{r e f} \\
n & =\frac{D_{L}}{D_{M}}
\end{aligned}
$$

where $n$ is the hydraulic actuator/pump displacement ratio.

Note that $\eta_{t M}$ and $\eta_{t L}$ suddenly decrease at near-zero speeds, resulting in static friction.

\section{Methods}

This section describes the proposed controller. The torque reference for the proposed controller is given by a proportional-derivative (PD) controller as follows:

$$
\begin{aligned}
\tau_{P D}^{r e f} & =m\left(K_{p}\left(\theta_{L}^{c m d}-\theta_{L}^{r e s}\right)+K_{v}\left(\dot{\theta}_{L}^{c m d}-\dot{\theta}_{L}^{r e s}\right)\right) \\
m & =\frac{\left(n^{2} \eta_{t L} m_{M}+m_{L}\right)}{n \eta_{t M} \eta_{t L}}
\end{aligned}
$$

where $\tau_{P D}^{r e f}$ is a torque reference calculated by the PD controller and $K_{p}$ and $K_{v}$ are the proportional and derivative gains, respectively. There are many controllers that use proportional-integral-derivative controllers to improve the steady-state performance. Instead, of these controllers, a DOB with the PD controller is implemented in this study because DOBs can powerfully suppress disturbances, eliminating the necessity of integral control $[9,10]$. Note that parameter tuning of DOBs is quite easy because the performance of the DOB can be designed using a simple parameter, cut-off frequency of a low-pass filter.

To limit the residual oscillation generated by the integrators of DOBs under large frictional forces, the estimation of the disturbance torque is suspended in the low-speed domain. We propose a new DOB as follows:

$$
\begin{aligned}
& \tau^{r e f}[k]=\tau_{P D}^{r e f}[k]+\hat{\tau}_{\text {mem }}^{\text {dis }}[k] \\
& \hat{\tau}_{\text {mem }}^{\text {dis }}[k+1]= \begin{cases}\hat{\tau}^{\text {dis }}[k+1] & \left.\left|\dot{\theta}_{L}\right|>T_{h 1}\right) \\
\hat{\tau}_{\text {mem }}^{\text {dis }}[k] & \left.\left|\dot{\theta}_{L}\right| \leq T_{h 1}\right),\end{cases}
\end{aligned}
$$

where $\tau^{r e f}$ and $\hat{\tau}^{\text {dis }}$ are a temporal torque reference and the estimated disturbance torque by the DOB, respectively. The suffix $[k]$ stands for a $k$ th value in the discrete time series. The method for determining $\hat{\tau}^{\text {dis }}$ is the same as in reference [9]. Values of $\hat{\tau}_{\text {mem }}^{\text {dis }}$ reflect $\hat{\tau}^{\text {dis }}$ values; updates are suspended when the velocity falls within the low-speed domain $\left|\dot{\theta}_{L}\right| \leq T_{h 1}$. Friction force overcompensation is avoided using this approach. In addition, as long as disturbances are step, $\hat{\tau}_{\text {mem }}^{\text {dis }}[k+1]=\hat{\tau}^{\text {dis }}[k+1]=\hat{\tau}^{\text {dis }}[k]=\hat{\tau}_{\text {mem }}^{\text {dis }}[k]$ holds at any time. Then, the memory hardly affects the tracking performance.

Ideally, $T_{h 1}$ is zero. However, because of computational errors of the angular velocity, the measured values may show a non-zero speed even if the actual motion stops. Therefore, $T_{h 1}$ should be the smallest value that is greater than the computational errors.

Frictional force that cannot be fully compensated for generates steady-state errors. We quantize $\tau^{r e f}$ such that the minimum output torque is greater than the maximum static friction. FMs-dynamic quantizers with a simple structure and a low pass filter-provide an easy and effective way to control the quantization errors. Note that system models are generally not required for FMs; this feature helps to ensure the robustness of the controller. Furthermore, some quantizers exhibit better performance with the explicit use of system dynamics in place of a system model $[15,16]$; however, the difference in performance is negligible despite the complexity of the compensator.

Figure 7 shows a schematic of an FM. In this diagram, $Q(s)$ satisfies $1-Q(s)=\left(\frac{T s}{T s+1}\right)^{2}$ and $\mathrm{S} / \mathrm{H}$ stands for "sample and hold," which describes the temporal resolution of the servomotor. $\tau_{C}^{r e f}$ is an intermediate torque reference and $\tau_{Q}^{r e f}$ is a quantized torque reference. Defining a time constant $T$ as $T=a S t$, with a sampling period $S t$ and a constant $a>1$, gives a pulse transfer function as follows:

$$
\begin{aligned}
Q(z) & =\frac{l_{1} z^{-1}+l_{2} z^{-2}}{1+l_{3} z^{-1}+l_{4} z^{-2}} . \\
l_{1} & =-\mathrm{e}^{-\frac{1}{a}}+\frac{1}{a} \mathrm{e}^{-\frac{1}{a}}+1, \\
l_{2} & =\mathrm{e}^{-\frac{2}{a}}-\frac{1}{a} \mathrm{e}^{-\frac{1}{a}}-\mathrm{e}^{-\frac{1}{a}}, \\
l_{3} & =-2 \mathrm{e}^{-\frac{1}{a}}, l_{4}=\mathrm{e}^{-\frac{2}{a}} .
\end{aligned}
$$

Note that as $a$ decreases, the quantization error also decreases. Using $Q(s), \tau^{r e f}$, the quantized torque 


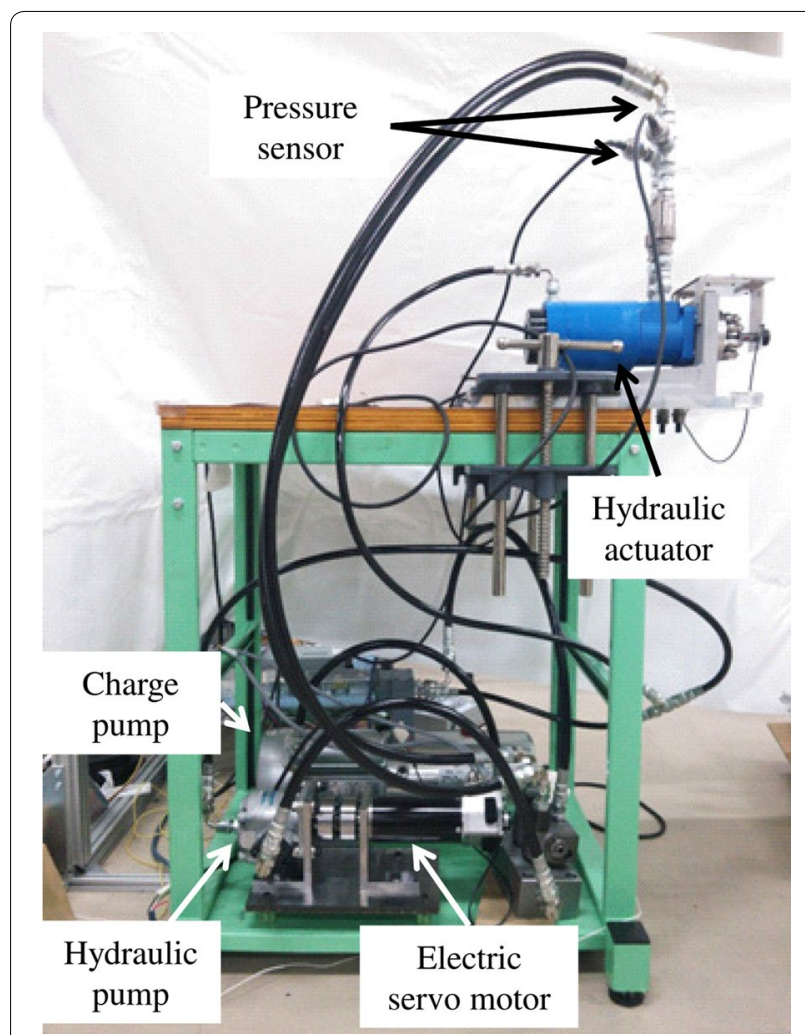

Fig. 6 Hydraulic system

of the servomotor $\tau_{Q}^{r e f}$, and the quantization error $\epsilon=\tau_{C}^{r e f}-\tau_{Q}^{r e f}$, the following relationship can be derived:

$$
\tau_{Q}^{r e f}=\tau^{r e f}+(1-Q(s)) \epsilon .
$$

In general, $\tau_{Q}^{r e f}$ can always be quantized. In this study, $\tau^{r e f}$ is quantized only in the low-speed domain to reduce the effect of quantization errors.

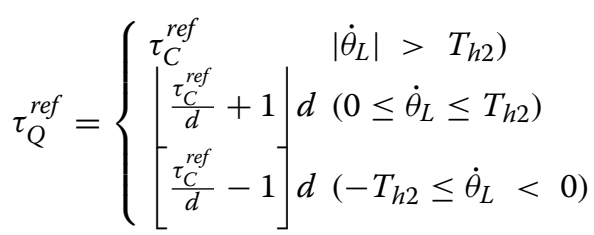

\lfloor\rfloor is a floor function where $d$ is a design parameter that should be greater than the maximum static frictional force. The second and third conditions reflect that $\tau_{Q}^{r e f}$ is free from static friction in the low-speed domain. Theoretically, $T_{h 2}$ can be arbitrary values that are greater than the static friction since quantization errors can be compensated for by the FM. However, a large $T_{h 2}$ results in harmonic distortion sounds of servomotors. Therefore, for practical implementation, designers should roughly identify the minimum velocity that is not in the static friction domain and choose a value of $T_{h 2}$ that is certainly greater than the identified value. Before torque efficiencies are reduced, $\tau_{C}^{r e f}$ should be quantized. The condition $T_{h 1}<T_{h 2}$ suppresses frictional effects.

Lastly, the current reference $I_{M}^{\text {ref }}$ is obtained as follows:

$$
I_{M}^{r e f}=\frac{\tau_{Q}^{r e f}}{K_{t}} .
$$

Figure 8 shows a comprehensive flow diagram for the proposed controller, where $g_{d}$ is the DOB cutoff frequency. "FM" stands for the block diagram of the FM as shown in Fig. 7.

Linear disturbances such as gravity are compensated for by the DOB while nonlinear static friction is compensated for by the FM. To avoid overcompensation of the integrator in the DOB, the update of the compensation value is suspended at low speeds. Then, designers can treat EHAs as if they are simple second-order systems without any disturbances resulting in an easy-to-tune controller.

High-frequency switching may harm the servomotor and hydraulic pump; a large $T$ should be selected to prevent damage. As a trade-off, control performance is degraded. If the control bandwidth is far broader than that of other controllers, FMs guarantee stability for closed-loop systems [17]. This assumption can usually be assured. For example, the FM bandwidth in this study was more than 100 times broader than that of other controllers, the PD controller and the DOB. In the experiment, the bandwidths of the PD controller and the DOB were set to 10 and $20 \mathrm{rad} / \mathrm{s}$, respectively, while the bandwidth of the $\mathrm{FM}$ was $5980 \mathrm{rad} / \mathrm{s}$.

\section{Results and discussion}

The effectiveness of the proposed controller was experimentally verified in this study. Table 1 shows the parameters used in this experiment. Before the primary experiment was conducted, the static friction of the EHA was initially measured.

The input torque was gradually increased, and we checked the value when the robot started moving. We

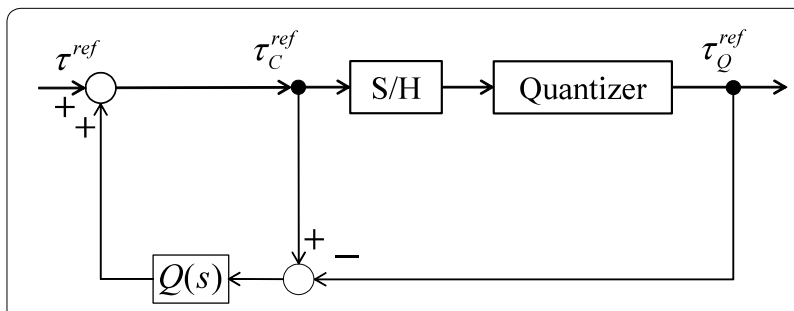

Fig. 7 Feedback modulator 
Table 1 Parameters

\begin{tabular}{lll}
\hline$m$ & Equivalent inertia & $3.3 \mathrm{kgm}^{2}$ \\
$K_{p}$ & Proportional gain & $100 / \mathrm{s}^{2}$ \\
$K_{v}$ & Derivative gain & $20 / \mathrm{s}$ \\
$K_{t}$ & Torque constant & $0.147 \mathrm{Nm} / \mathrm{A}$ \\
$g_{d}$ & Cut-off frequency of DOB & $20 \mathrm{rad} / \mathrm{s}$ \\
$S t$ & Sampling period & $0.001 \mathrm{~s}$ \\
$a$ & Time constant ratio & 1.05 \\
$D_{1}$ & Displacement of pump & $3.08 \mathrm{~cm}^{3} / \mathrm{rev}$ \\
$D_{2}$ & Displacement of motor & $371 \mathrm{~cm}^{3} / \mathrm{rev}$ \\
$d$ & Quantization & $70 \mathrm{Nm}$ \\
$T_{h 1}$ & Threshold & $0.00223 \mathrm{rad} / \mathrm{s}$ \\
$T_{h 2}$ & Threshold & $0.1 \mathrm{rad} / \mathrm{s}$ \\
$\eta_{t M}$ & Nominal torque efficiency & 1 \\
$\eta_{t L}$ & Nominal torque efficiency & 1 \\
\hline
\end{tabular}

found that the static friction did not exceed $70 \mathrm{Nm}$; $d$ was set to $70 \mathrm{Nm}$.

\section{Performance comparison}

In this experiment, step and sinusoidal commands were given to the proposed controller and two conventional controllers. The two conventional controllers included a PD controller and the following: (1) a DOB with a control input given by $I_{M}^{r e f}=\frac{1}{K_{t}}\left(\tau_{P D}^{r e f}+\hat{\tau}^{d i s}\right)$ and (2) a DOB and a dither signal with a control input given by $I_{M}^{r e f}=\frac{1}{K_{t}}\left(\tau_{P D}^{r e f}+\hat{\tau}^{\text {dis }}+\tau^{\text {dither }}\right)$. The dither signal, $\tau^{\text {dither }}=A \sin (10 \pi t)$, is intended to suppress static friction. Note that the frequency of the dither signal was determined according to reference [14]. Figures 9 and 10 show the experimental results with step and sinusoidal (of $0.5 \mathrm{~Hz}$ ) commands, respectively. Figures 9a and 10a show the results for the PD controller with the DOB. Figures $9 \mathrm{~b}, \mathrm{c}$ and $10 \mathrm{~b}, \mathrm{c}$ show the results for the PD controller with the DOB and dither signal, with dither signal amplitudes of 5.0 and 50.0. The amplitudes were determined so that one of them was lower than the static friction and the other was greater than the static friction. Since one with a greater value still had a value that was lower than the quantization value of the proposed method $(70 \mathrm{Nm})$, the validity of the proposed method can be confirmed with respect to the two dither signals. Figures $9 \mathrm{~d}$ and $10 \mathrm{~d}$ show the results for the proposed controller. Figure 9e shows the result for the PD controller with the FM when the DOB was not used. Figure of shows the result for the PD controller with the proposed DOB given in (7) when the FM was not used. The red lines indicate the commands; the green lines show the responses.

As shown in Fig. 9a, residual oscillation was generated for the PD controller with the DOB, indicating difficulty when using integrators under a large static frictional force. Figure $9 \mathrm{~b}$ shows that small-amplitude dither signals have almost no compensatory effect on the static friction. Figure 9c shows that large-amplitude dither signals lead to oscillatory responses. Without an accurate estimation of static friction, good control performance cannot be obtained by feedforward controllers. In Fig. 9e, because there was no integrator in the controller, residual oscillation was not generated while large steady-state errors were found due to the lack of a DOB. In Fig. 9f, there was no residual oscillation since the disturbance estimation was suspended at low speeds. However, small steadystate errors caused by static friction were found. Comparatively, residual oscillation was almost fully suppressed in the proposed controller, while steady-state errors were almost suppressed, as shown in Fig. 9d. Even though the quantization value, $d$, was not strictly designed to exceed the static friction, the frictional effects were negligible when the actual value was approximately $24 \mathrm{Nm}$.

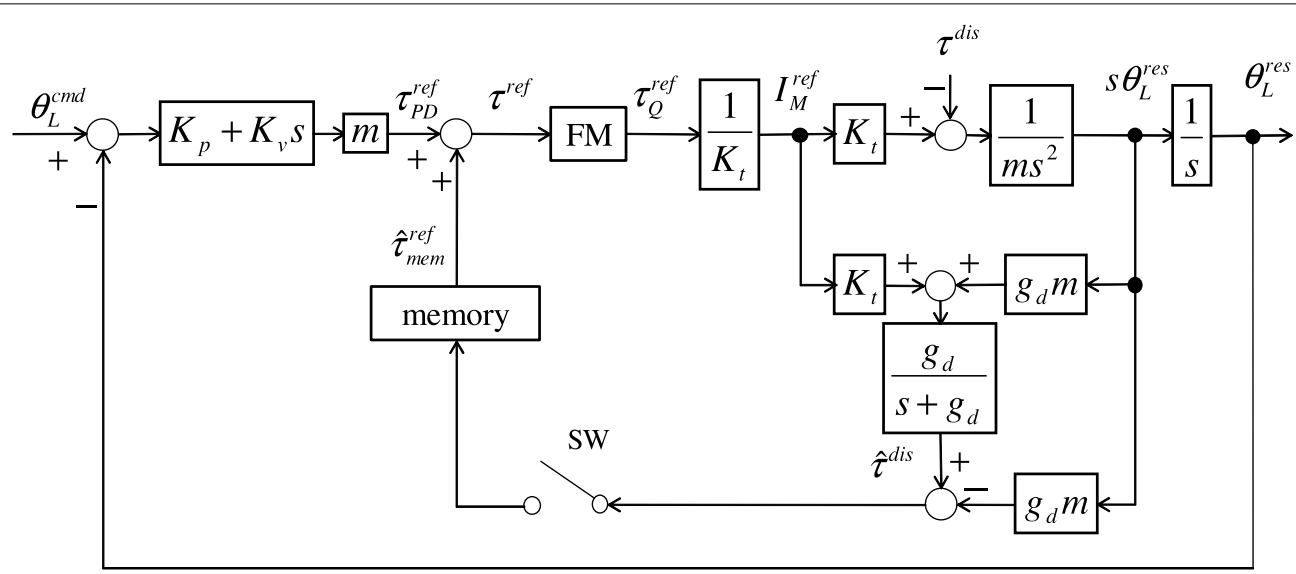

Fig. 8 Block diagram of the proposed method 


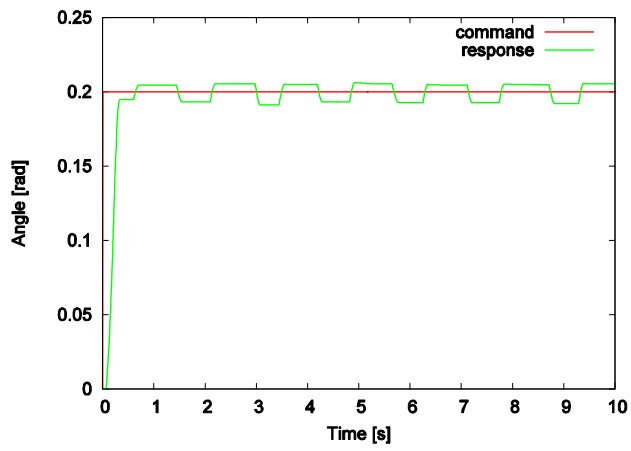

a Disturbance observer

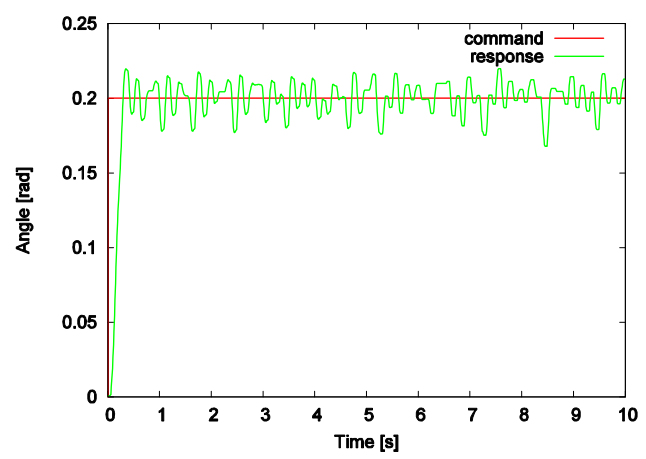

c Disturbance observer + dither signal $(A=50)$

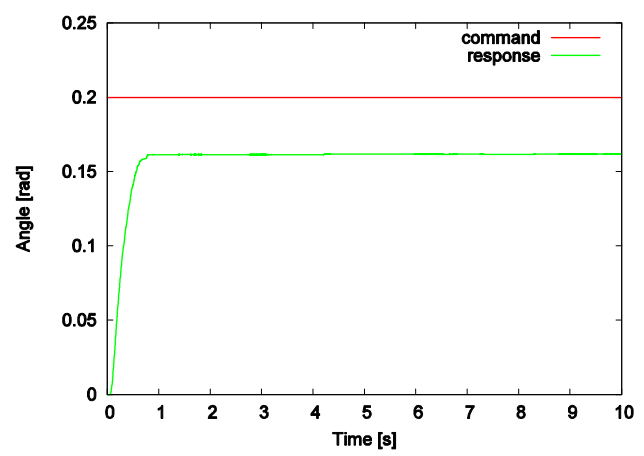

e Feedback modulator

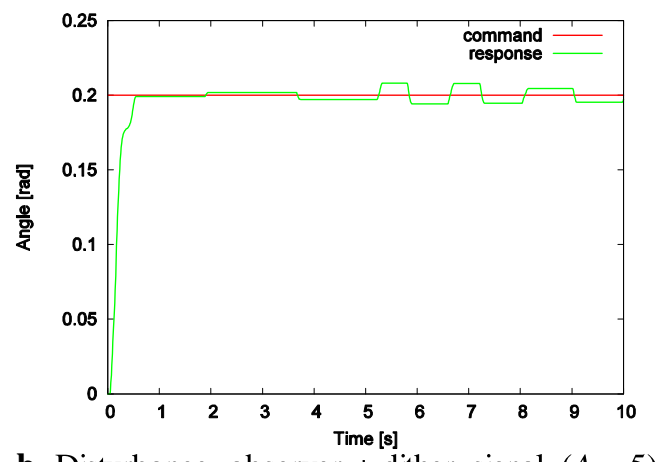

b Disturbance observer + dither signal $(A=5)$

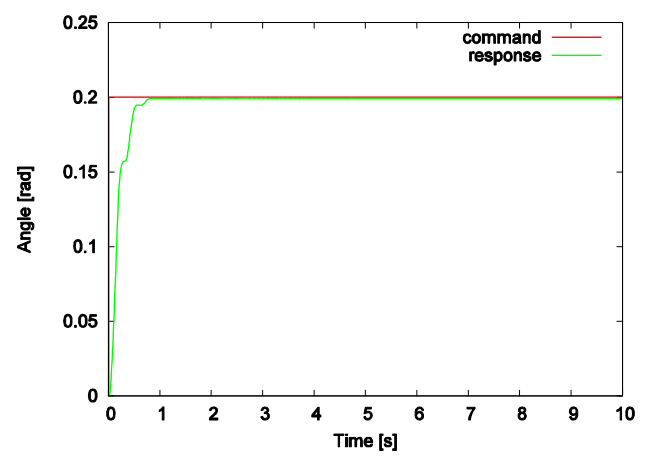

d Disturbance observer with memory + Feedback modulator

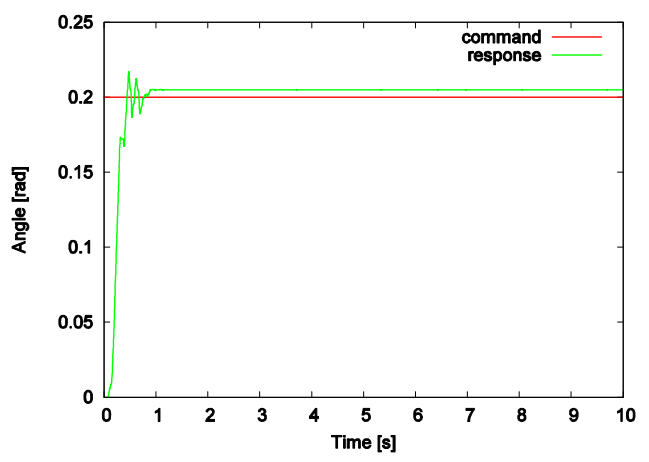

f Disturbance observer with memory

Fig. 9 Experimental results (step response)

Differences among controller design were less apparent when considering the sinusoidal wave responses in Fig. 10a, b and d. The three controllers had almost the same performance in tracking sinusoidal waves because the effect of the static friction was not large for sinusoidal movement. In addition, owing to the overcompensation of friction, Fig. 10c shows larger tracking errors. Figure 11 shows the frequency characteristics of the three controllers: the PD controller with the DOB, the PD controller with the $\mathrm{DOB}$ and the dither signal $(A=5)$, and the proposed method. As we can see from the figures, the characteristics were quite similar. 


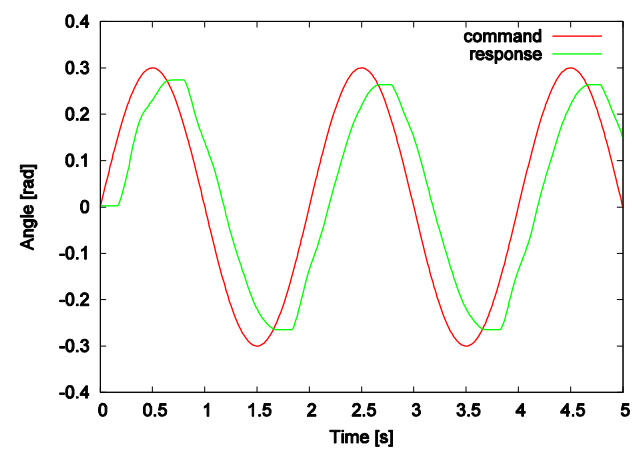

a Disturbance observer

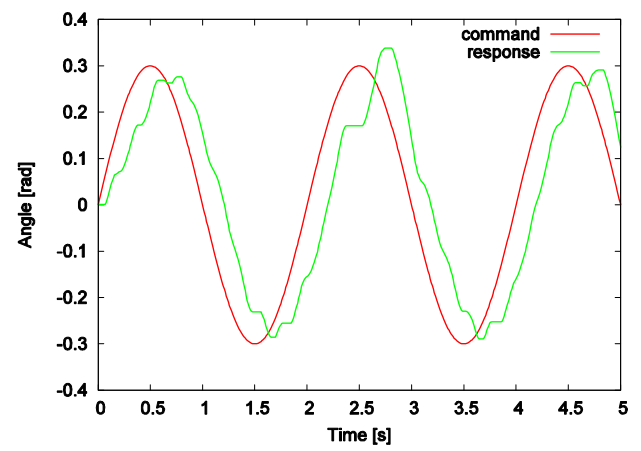

c Disturbance observer + dither signal $(A=50)$

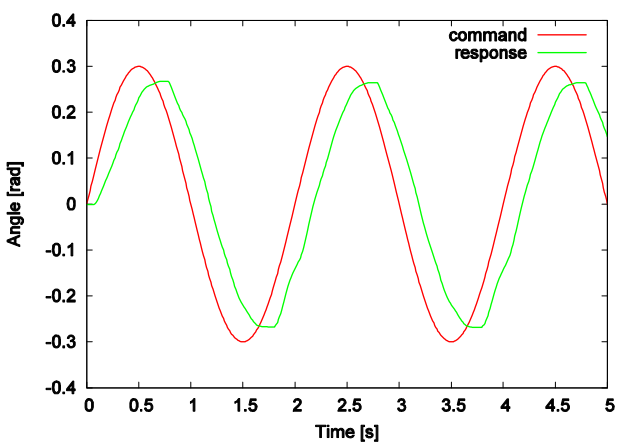

b Disturbance observer + dither signal $(A=5)$

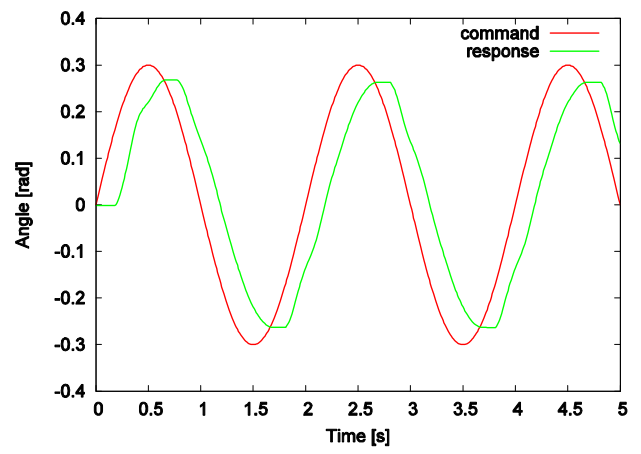

d Disturbance observer with memory + Feedback modulator

Fig. 10 Experimental results (sinusoidal wave $0.5 \mathrm{~Hz}$ )

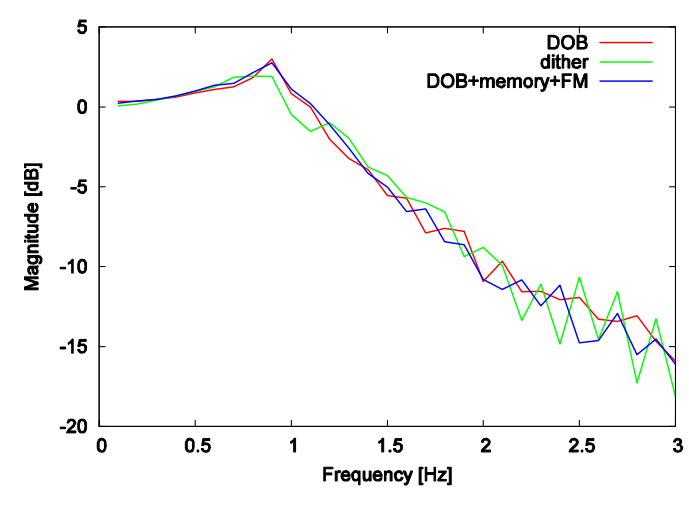

a Magnitude

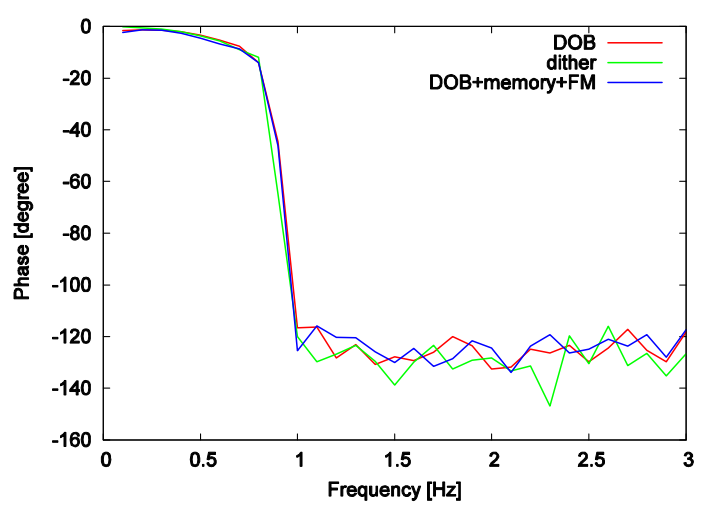

b Phase

Fig. 11 Bode diagram

Figure 12 shows the control inputs, with a and b representing inputs for the PD controller and the DOB and c and $\mathrm{d}$ representing inputs for the proposed controller. As shown in Fig. 12a, the input for the PD controller and the DOB was iteratively integrated to reach a maximum static friction of approximately $24 \mathrm{Nm}$. When the input exceeded the maximum static friction, an overshoot response was initiated, and the input was again integrated to reach the maximum static friction. As shown in Fig. 12b, no static friction effects were observed for the same conventional controller in sinusoidal movements. As shown in Fig. 12c and $\mathrm{d}$, the proposed controller effectively quantized inputs and static friction effects were suppressed. Furthermore, as shown in Fig. 12d, the proposed controller's input was 


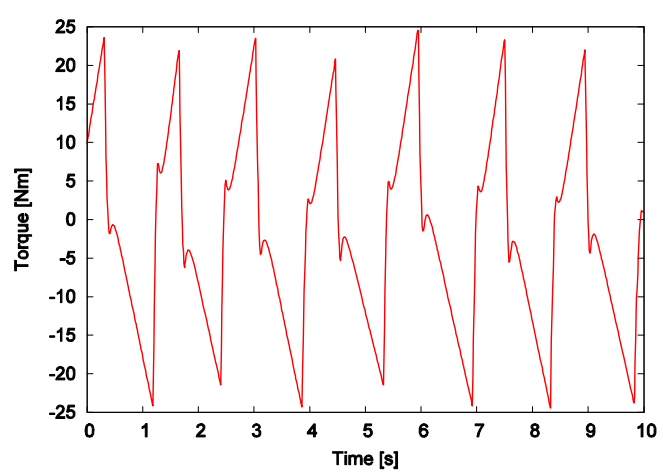

a Disturbance observer (step)

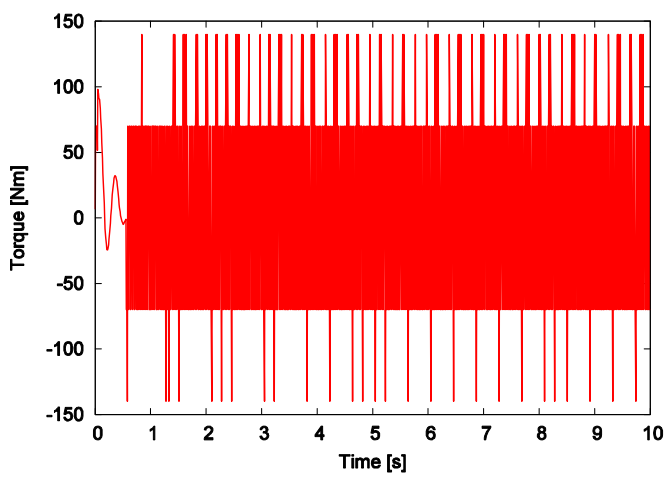

c Disturbance observer with memory + Feedback modulator (step)

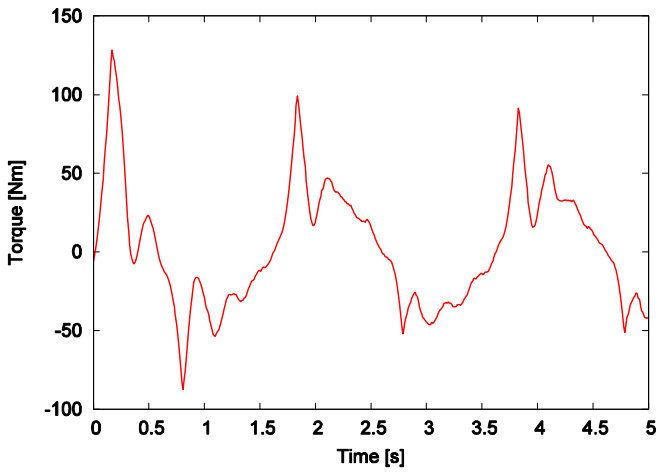

b Disturbance observer $(0.5 \mathrm{~Hz})$

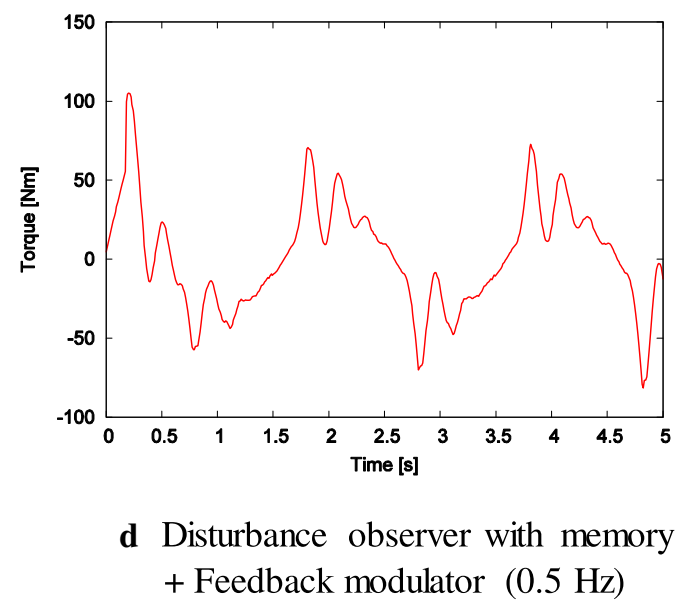

Fig. 12 Torque reference

similar to the conventional controller's waveform input when the actuator did not experience static friction. Therefore, we can summarize that the proposed controller exhibited better performance at low speeds and comparable performance at higher speeds when compared to conventional controllers. In other words, the proposed controller can improve the performance at low speeds without degrading the performance at high speeds.

\section{Conclusions}

Electro-hydrostatic actuators offer faster control speeds but suffer from higher static friction levels when compared with open hydraulic circuits. Therefore, EHAs require adequate controllers to compensate for the effects of static friction. In this study, FMs and DOBs were integrated to suppress the effects of static friction in EHAs. FMs guarantee precise and stable input quantization. As long as the input frequencies are far greater than the natural frequencies of the systems, quantization has little effect on the output. In this study, steady-state errors caused by linear disturbances were suppressed by the
DOB and the residual oscillation caused by static friction was suppressed by the FM. The integration was achieved using only two intuitive tuning parameters, $T_{h 1}$ and $T_{h 2}$; strict parameter tuning was not required. Furthermore, system models are not required for FM implementations, simplifying efforts in enhancing robustness. Hydraulic actuators have been associated with significant nonlinearities that challenge controller design. In this study, we proved that the nonlinearities do not have to be problematic. Using the proposed design, others can develop simple and easy-to-tune linear controllers for hydraulic actuator implementation, subsequently increasing the use and utility of hydraulic actuators.

We succeeded in improving the low speed performance while performance in high frequency domain has not been improved. The control bandwidth was still less than $1 \mathrm{~Hz}$. We recently revealed that with sufficient friction compensation, EHAs actually have the characteristics of a two-mass resonant system. Then, the next target will be performance improvement in the high-speed domain by suppressing the resonance using FMs and DOBs. 


\section{Authors' contributions}

SS drafted the manuscript, participated in the design of the study, and carried out part of the studies. TT participated in its design and coordination. Both authors read and approved the final manuscript.

\section{Acknowledgements}

This study was supported in part by a Grant-in-Aid for Scientific Research (C) (16K06410).

\section{Competing interests}

The authors declare that they have no competing interests.

Received: 22 September 2016 Accepted: 6 December 2016

Published online: 03 January 2017

\section{References}

1. Kaminaga H, Ono J, Nakashima Y, Nakamura Y (2009) Development of backdrivable hydraulic joint mechanism for knee joint of humanoid robots. In: Proceedings of the 2009 IEEE international conference on robotics and automation, pp 1577-1582

2. Mohanty A, Yao B (2011) Integrated direct/indirect adaptive robust control of hydraulic manipulators with valve deadband. IEEE/ASME Trans Mechatron 16(4):707-715

3. Dasmahapatra S, Sarkar BK, Saha R, Chatterjee A, Mookherjee S, Sanyal D (2015) Design of an adaptive fuzzy-bias smc and validation for a rugged electrohydraulic system. IEEE/ASME Trans Mechatron 20(6):2708-2715

4. Ho TH, Ahn KK (2012) Speed control of a hydraulic pressure coupling drive using an adaptive fuzzy sliding-mode control. IEEE/ASME Trans Mechatron 17(5):976-986

5. Eaton Industries Ltd. http://www.eaton.jp/EatonComJapan/. Accessed 25 Dec 2015
6. Guan C, Pan S (2008) Nonlinear adaptive robust control of single-rod electro-hydraulic actuator with unknown nonlinear parameters. IEEE Trans Control Syst Technol 16(3):434-445

7. Yao J, Deng W, Jiao Z (2015) Adaptive control of hydraulic actuators with lugre model based friction compensation. IEEE Trans Ind Electron 62(10):6469-6477

8. Ohgi T, Yokokohji Y (2008) Control of hydraulic actuator systems using feedback modulator. J Robot Mechatron 20(5):695-708

9. Ohnishi K, Shibata M, Murakami T (1996) Motion control for advanced mechatronics. IEEE/ASME Trans Mechatron 1(1):56-67

10. Lee IY, Kim TH, Choi SR (2008) Hydraulic servo system using a feedback linearization controller and disturbance observer-sensitivity of system parameters. In: the 7th JFPS international symposium on fluid power, pp 307-312

11. Won D, Kim W, Shin D, Chung CC (2015) High-gain disturbance observerbased backstepping control with output tracking error constraint for electro-hydraulic systems. IEEE Trans Control Syst Technol 23(2):787-795

12. Yao J, Jiao Z, Ma D (2014) Extended-state-observer-based output feedback nonlinear robust control of hydraulic systems with backstepping. EEE Trans Ind Electron 61(11):6285-6293

13. Sakaino S, Tsuji T (2014) Integration of disturbance observer and feedback modulator for dead zone compensation of hydraulic actuator. In: Proceedings of the IEEE 40th annual conference of the IEEE industrial electronics society, pp 2786-2791

14. Phuong TT, Ohishi K, Mitsantisuk C (2014) Fpga-based high-performance force control system with friction-free and noise-free force observation. EEE Trans Ind Electron 61(2):994-1008

15. Azuma S, Sugie T (2008) Synthesis of optimal dynamic quantizers for discrete-valued input control. IEEE Trans Autom Control 53(9):2064-2075

16. Minami Y, Azuma S, Sugie T (2009) Multirate-sampling dynamic quantizers for non-minimum phase linear control systems. SICE J Control Meas Syst Integr 2(4):229-235

17. Ishikawa M, Maruta I, Sugie T (2006) Quantized controller design using feedback modulators. Trans Soc Instrum Control Eng 43(1):31-36 in Japanese

\section{Submit your manuscript to a SpringerOpen ${ }^{\odot}$ journal and benefit from:}

- Convenient online submission

- Rigorous peer review

- Immediate publication on acceptance

- Open access: articles freely available online

- High visibility within the field

- Retaining the copyright to your article 